\section{SLEEP AND GENERAL HEALTH PREDICT HIGHER INJURY RATES IN ENDURANCE ATHLETES: A PROSPECTIVE STUDY}

${ }^{2}$ Kieran O'Sullivan, ${ }^{2,3}$ Richard Johnston, ${ }^{2}$ Roisin Cahalan, ${ }^{4}$ Laura Bonnett, ${ }^{1}$ Mark Maguire ${ }^{1}$ Phil Glasgow, ${ }^{5}$ Sharon Madigan, ${ }^{2}$ Thomas M Comyns. ${ }^{1}$ Irish Rugby Football Union, Dublin, Ireland; ' University of Limerick, Limerick, Ireland; ${ }^{3}$ La Trobe Sport and Exercise Medicine Research Centre, School of Allied Health, La Trobe University", Melbourne, Australia; ${ }^{4}$ Department of Biostatistics, University of Liverpool, Liverpool, UK; ${ }^{5}$ Sport Ireland Institute, National Sports Campus, Dublin, Ireland

\subsection{6/bjsports-2021-IOC.54}

Background Rates of injury among endurance sporting participants are high, as are subjective health complaints (SHCs - e. g. poor sleep, low mood). However, there is limited prospective data evaluating the relationship between SHCs and rates of new injury.

Objective To examine longitudinal associations between SHCs, sleep quantity and new injury within an endurance sport population.

Design Prospective cohort study.

Setting Competitive, sub-elite, endurance sports.

Patients (or Participants) Ninety-five endurance sporting participants recruited from running, triathlon, swimming, cycling and rowing disciplines. $92.6 \%$ of 95 participants submitted data for all 52 weeks, with the remainder completing $\geq 30$ weeks.

Main Outcome Measurements Participants submitted weekly data regarding SHCs (cardiorespiratory, gastrointestinal and psychological/lifestyle), sleep quantity, training load and new injury episodes. Applying a 7- and 14-day lag period, a shared frailty model was used to explore new injury risk associations with total SHCs and sleep quantity, as planned a-priori.

Results Seven-day lag psychological/lifestyle SHCs were significantly associated with new injury risk (Hazard ratio (HR) $=1.32$; CI $95 \%=1.01-1.72, \mathrm{p}<0.04)$. Whilst cardiorespiratory $(\mathrm{HR}=1.15$; CI $95 \%=0.99-1.36, \mathrm{p}=0.07)$ and gastrointestinal $(\mathrm{HR}=0.77$; CI 95\%=0.56-1.05, $\mathrm{p}=0.09)$ SHCs were not significantly associated. New injury risk had a significant increased association with 14 -day lag $<7 \mathrm{hrs}$ /day sleep quantity $(\mathrm{HR}=1.51$; CI $95 \%=2.02-1.13, \mathrm{p}<0.01)$ and a significant decreased association with $>7 \mathrm{hrs} /$ day sleep quantity $(\mathrm{HR}=0.63$, CI 95\% $=0.45-0.87, \mathrm{p}<0.01$. A secondary regression analysis demonstrated no significant association with total SHCs and training load factors (Relative Risk $(\mathrm{RR})=0.08$, CI $95 \%=0.04-0.21, \mathrm{p}=0.20)$.

Conclusions To minimise an increased risk of new injuries within an endurance sporting population, this study demonstrates that psychological/lifestyle SHCs and sleep quantity should be considered. The study also highlights a lag period between low sleep quantity and its subsequent impact on new injury risk. No association was demonstrated between subjective health complaints, sleep quantity and training load factors.

\section{INJURY, ILLNESS AND COACHING STYLE: ASSOCIATIONS WITH BURNOUT IN YOUNG ELITE ATHLETES}

${ }^{1}$ Nils Fredrik Holm Moseid, ${ }^{1}$ Christine Holm Moseid, ${ }^{2}$ Pierre-Nicolas Lemyre, ${ }^{1}$ Morten Wang Fagerland, ${ }^{1}$ Roald Bahr. 'Oslo Sports Trauma Research Center, Department of Sports Medicine, The Norwegian School of Sport Sciences, Oslo, Norway; ${ }^{2}$ Department of Coaching and Psychology, The Norwegian School of Sport Sciences, Oslo, Norway

10.1136/bjsports-2021-IOC.55
Background Previous research indicates that psychological factors is associated with injury incidence. The associations between health burden, psychological health and coaching style have, to our best knowledge not been investigated.

Objective Explore associations between health burden, needsupportive coaching and athlete burnout after three years in elite sports high school.

Design Mixed prospective and retrospective cohort study. We collected health data for 124 weeks, the first 26 weeks prospectively, the remaining 98 weeks retrospectively by interview. Participants completed a web-based survey at the end of the study period.

Setting Three Norwegian High School Sport Academies.

Patients (or Participants) 210 students, of which 202 graduated after three years, 8 dropped out before graduation.

Interventions (or Assessment of Risk Factors) We measured health burden using The Oslo Sports Trauma Research Centers Questionnaire on health problems, results are presented per 1000 arbitrary units. Participants completed the 'The Supportive Coach' (SC), a ten-item questionnaire that measures the athlete's perceived support from the coach in the psychological domains of autonomy, competence and relatedness, on a 1-5 scale. We used linear regression for statistical analysis.

Main Outcome Measurements The Athlete Burnout Questionnaire (ABQ) score. ABQ measures symptoms of athlete burnout on a $1-5$ scale.

Results Average ABQ-score was 2.3 (mean, 95\% confidence interval: 2.2 to 2.4 ). Average yearly health burden was 0.96 (median, range 0.03-6.9). Average SC-score was 4.0 (median, range: 1.3 to 5.0 ). Health burden, adjusted for coaching style and major life events, was associated with increasing symptoms of burnout (B: $0.1,0.09$ to $0.21, p<0,001)$. Need-sup portive coaching is associated with decreasing symptoms, (B: $0.24,-0.35$ to $-0.14, \mathrm{p}<0.001)$.

Conclusions An increasing health burden is associated with increasing symptoms of athlete burnout. A need-supportive coach is associated with decreasing symptoms. Coaches should be aware of symptoms of burnout in ill and injured athletes, and consider using a need-supportive approach.

\section{OLYMPIC-CAREER RELATED SPORTS INJURY EPIDEMIOLOGY: THE RETIRED OLYMPIAN MUSCULOSKELETAL HEALTH STUDY (ROMHS)}

${ }^{1}$ Debbie Palmer, ${ }^{2}$ Dale Cooper, ${ }^{3}$ Carolyn Emery, ${ }^{4,5}$ Mark Batt, ${ }^{6,7}$ Lars Engebretsen, ${ }^{5,8}$ Brigitte Scammell, ${ }^{7}$ Torbjørn Soligard, ${ }^{6}$ Kathrin Steffen, ${ }^{3,9}$ Jackie Whittaker, ${ }^{7}$ Richard Budgett. ${ }^{1}$ Institute of Sport, PE and Health Sciences, Moray House School of Education and Sport, University of Edinburgh, Edinburgh, UK; ${ }^{2}$ School of Allied Health Professions, University of Leicester, Leicester, UK; ${ }^{3}$ Sport Injury Prevention Research Centre, Faculty of Kinesiology, University of Calgary, Calgary, Canada; ${ }^{4}$ Nottingham University Hospitals Trust, Nottingham, UK; ${ }^{5}$ Centre for Sport, Exercise and Osteoarthritis Versus Arthritis, Queens Medical Centre, Nottingham, UK; ${ }^{6}$ Department of Sports Medicine, Oslo Sports Trauma Research Centre, Norwegian School of Sport Sciences, Oslo, Norway; ${ }^{7}$ Medical and Scientific Department, International Olympic Committee, Lausanne, Switzerland; ${ }^{8}$ School of Medicine, University of Nottingham, Nottingham, UK; ${ }^{9}$ Department of Physical Therapy, University of British Columbia, Vancouver, Canada

\subsection{6/bjsports-2021-IOC.56}

Background There are numerous studies describing elite athlete injury patterns seasonally and during major sporting events, however little is known about injury patterns during an elite athlete's entire sporting career. 